\title{
BEYOND RE-ENGINEERING: \\ DE-ENGINEERING THE CORPORATION
}

\author{
René Pellissier ${ }^{1}$ \\ and \\ Paul Kruger ${ }^{2}$
}

\begin{abstract}
This paper addresses the failure of re-engineering to deliver its promise of better organizational performance through radical change. It looks at the change in Hammer and Champy's own perceptions of what re-engineering really is and studies reasons for its failure - the problem of ubiquitous IT, the problem of process integration and re-engineering's radical Marxist connotations. Newton's linear clock-like machine view of the world is seen as part of the failure of re-engineering to deliver and is replaced by a quantum view of continued, evolutionary change. Consequently, the chaos phenomenon is studied, especially with respect to the principle of self-organization. Lastly, the proposition of (evolutionary) de-engineering the organization as clean up after or beyond (revolutionary) re-engineering is discussed following on the principles of chaos theory and the emerging viewpoint of the organization as a living entity.
\end{abstract}

\section{KEY WORDS}

Re-engineering, de-engineering, Newtonian science, self-organization, IT, information, process integration, Marxism, linear thinking, non-linearity, quantum theory, Heisenberg's Uncertainty Principle.

${ }^{1}$ Graduate School for Business Leadership, University of South Africa ${ }^{2}$ Department of Industrial and Systems Engineering, University of Pretoria. 
'Wisdom is about living harmoniously in the universe,

which is itself a place of order and justice

that triumphs over chaos and

employs chance for its ultimate purpose.'

Matthew Fox

\section{INTRODUCTION TO RADICAL CHANGE}

Societal concerns (for instance, fear of nuclear events, the thinning of the ozone layer and quality of food and water) are exacerbated for businesses by added pressures like downsizing, restructuring and the chaotic nature of the capital markets. There is pressure from global competitors in a once secure domestic market. This is underscored by new commercial arrangements, with American capitalists interacting with Japanese and German models, where command economies more oriented toward free markets fuse to create one world economy consisting of multiple markets. The focus of trade has migrated from the Atlantic to the Pacific. Fluctuating inflation causes a questioning of values. There is a transition to another epoch. The world is leaving the existing era for a new stage in history. The primary lesson to be learnt from history is this: Periodically, like Toffler's economic waves [1], society needs a sharp break with old habits. It needs deliberately to learn new ways of existing and doing business. Leadership should see that simply 'pressing the pedal harder' by doing more of the same does not work, nor do cosmetic changes according to the 'flavour of the month', nor do piecemeal solutions. Businesses need a radical redesign and total rethink of the ways in which they do their business.

In 1990, Hammer and Champy changed forever the scene for companies who were trying to reinvent themselves because the environment and their customers said that they should, through the introduction of the concept of re-engineering the business processes, when they wrote [2: p32]: 'Re-engineering involves the fundamental rethink and radical redesign of business processes to achieve dramatic improvements in critical, contemporary measures of performance such as cost, quality service and speed.' While, according to Hamel and Prahalad [3: p12]: 'Any company that is more successful at restructuring than re-engineering will find itself getting smaller faster than it is getting better'. The above summarises the (general) consensus and concerns with regard to the misconceptions of reengineering and its confusion with downsizing and restructuring. Furthermore, the ubiquitous information technology (IT) becomes the critical enabler and facilitator in their re-engineering, when Hammer and Champy contend that: 'A Company that cannot change the way it thinks about IT, cannot re-engineer. A company that equates technology with automation cannot re-engineer.' [2: p83]. Their proposition of replacing a lacklustre diamond by a sparkling one suggests that IT forms an integral part of the new way of working. They believe 'state of the art IT.' to be 'an essential enabler' in corporations re-inventing themselves and continue to cite examples of the misuse of IT in the organization of work [2: p101].

Hammer and Champy [4] discuss the simple shift beyond re-engineering. They retain the original definition of re-engineering but the emphasis shifts from the word 'radical' (connoting clean sheet design) to 'processes' (representing the aspect of the organization that is redesigned). They believe it 
should be recognised that there are two distinct but related ideas - i.e. aligning the organization around processes and instituting major changes in how processes operate. In this context, they believe that the ratio of workers to managers could double. (The latter contradicts the conception that automation (using technology) can decrease the numbers of workers.) They maintain re-engineering is not any of the following: Downsizing, automation, restructuring, reorganising, debureaucratisation, delayering, total quality management or continuous improvement, or mass customisation. Hammer himself subsequently presents a different viewpoint [5]. He contends that many organizations misinterpreted the message and used re-engineering as an excuse to slash employee numbers. He strongly suggests that, after an organization has trimmed off the fat, they should re-engineer for growth. He goes on to say that organizations should follow through by focusing on products, customers and market share going beyond re-engineering. This proposes an evolution of re-engineering as business evolves with it. Gurus are unanimous that the problem with re-engineering lies in its unflinching focus on the bottom line [6]. They believe that redesigning processes as a cost-cutting measure is counterproductive since a lot of valuable knowledge and information are simply discarded. The fact that Hammer himself is reengineering re-engineering proves this more than anything else could.

Organizations up to now have been focusing their attention on structure and organizational design, on gathering extensive numerical data and on making decisions using sophisticated mathematical and financial ratios. The essence of good management was building elaborate business models, contemplating more and more variables - thus creating more advanced forms of analysis. Thus, management really believed that one could study the parts (no matter how many of them there were) in order to arrive at knowledge of the whole. Moreover, most of the management tools available today reduce, describe and separate things into cause and effect and draw the world in lines and boxes. However, it is contended that such a world based on machine images, is also a world filled with boundaries. In other words, these management paradigms create roles and accountabilities, drawing lines of authority and limits to responsibility - even around the flow of experience, thus shaping business thinking of reality in terms of variables. As managers, we study variables for their alleged dependent and independent properties, treating them as separate, as well-behaved and as well-bounded. Information is portrayed as separate, measurable and integer-valued entities, i.e. market share, number of defectives.

\section{WHAT WENT WRONG WITH RE-ENGINEERING?}

\subsection{Process-focused re-engineering}

Re-engineering as management fad, is well advanced in its cycle. Intended to boost competitiveness through simpler, leaner, more productive processes, re-engineering is rampant in labour- and capitalintensive industries (such as cars, telecommunications, drugs and aerospace); it has spread to the service sector, particularly insurance and banking. By all accounts, re-engineering is in trouble. According to one American survey, companies will spend $\$ 52$ billion on business re-engineering, of which $\$ 40$ billion will go towards IT. It seems that organizations are not satisfied with the reengineering label [7].

It seems unusual that the first assessment of re-engineering comes from a leading re-engineering consultancy, namely, the CSC Foundation Index. Based on a survey of 497 large companies in the 
Hammer and Champy [4] describe re-engineering as '.. the idea of reunifying (previously: breaking down) those tasks into coherent business processes.' They go on to write that re-engineering rejects Adam Smith's industrial paradigm - the division of labour, economies of scale and hierarchical control. However, they still fail to provide a more useful definition of re-engineering than the one presented in 1990. They maintain that their definition contains four key words, namely 'fundamental', 'radical', 'process' and 'dramatic'. However, it is suggested that there really is only one key word, namely process. The essence of re-engineering has little to do with the other three, but totally focuses on reintegrating the process - in terms of tasks, labour and knowledge. This will be explained in the table below.

\section{Table 1: The main focus of re-engineering - reintegration of processes}

\begin{tabular}{|c|c|}
\hline TRITITSS: & 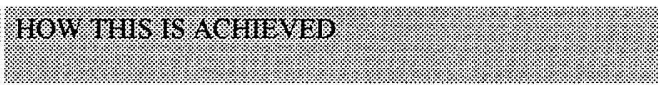 \\
\hline Reintegration of tasks & $\begin{array}{l}\text { Combine snaller processes into larger integrated units } \\
\text { Reduce number of parts in products and processes. }\end{array}$ \\
\hline Rerntegrtilon of laboin: & 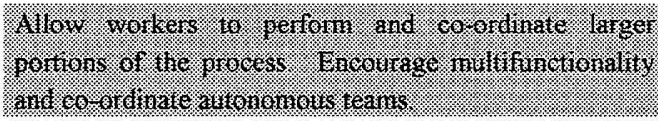 \\
\hline Reintegration & Workers must know larget portions (not smaller ones) \\
\hline
\end{tabular}

Source: $[10]$.

Thus, the re-engineering solution deals with at least three separate and relatively independent and differentially manageable aspects, namely division of tasks, division of labour and division of knowledge. Zeleny [10] likens the existence of re-engineering to the example of the move from horse carriage to combustion engine. Continuously and incrementally improving the components of the horse carriage has limited scope and is useful only in preserving the status quo. Re-engineering the horse carriage (fundamentally, radically and dramatically) is not enough. The focus has to be on the reintegration of task, labour and knowledge of the product or service. This is also true for management systems. The task is not to improve a hierarchical, centralised command system (fundamentally, radically and dramatically), but to re-integrate the processes of autonomous process-owners.

The following are generally cited in literature as reasons why re-engineering projects fail:

(i) Unclear definitions: re-engineering is more than automation or re-organization although it almost always effects organizational change. It goes beyond TQM seeking breakthrough measures of performance, pursuing multifaceted improvement goals, for instance quality, cost, flexibility, speed, accuracy and customer satisfaction - concurrently and with little trade off.

(ii) Unrealistic expectations: One of the consequences of (i) above, is the over-optimistic viewpoint about the domain of re-engineering.

(iii) Inadequate resources: Adequate resourcing of the re-engineering is a balanced mix of insiders and outsiders for the re-engineering. The question of IT resources also plays a part in this. 
Hammer and Champy [4] describe re-engineering as ".. the idea of reunifying (previously: breaking down) those tasks into coherent business processes.' They go on to write that re-engineering rejects Adam Smith's industrial paradigm - the division of labour, economies of scale and hierarchical control. However, they still fail to provide a more useful definition of re-engineering than the one presented in 1990. They maintain that their definition contains four key words, namely 'fundamental', 'radical', 'process' and 'dramatic'. However, it is suggested that there really is only one key word, namely process. The essence of re-engineering has little to do with the other three, but totally focuses on reintegrating the process - in terms of tasks, labour and knowledge. This will be explained in the table below.

\section{Table 1: The main focus of re-engineering - reintegration of processes}

\section{PRo. IESS: \\ REINTECR ATION}

Reintegration of tasks

10.100 .10

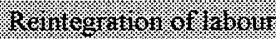

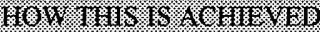

Combine snaller processes into latger integrated units Reduce number of parts in products and processes:

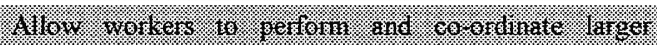

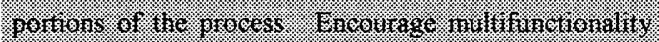

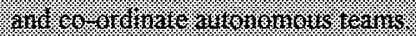

Reintegration knowledge of Workers nust know larget portions (not smaller ones) of the process and product:

Source: [10].

Thus, the re-engineering solution deals with at least three separate and relatively independent and differentially manageable aspects, namely division of tasks, division of labour and division of knowledge. Zeleny [10] likens the existence of re-engineering to the example of the move from horse carriage to combustion engine. Continuously and incrementally improving the components of the horse carriage has limited scope and is useful only in preserving the status quo. Re-engineering the horse carriage (fundamentally, radically and dramatically) is not enough. The focus has to be on the reintegration of task, labour and knowledge of the product or service. This is also true for management systems. The task is not to improve a hierarchical, centralised command system (fundamentally, radically and dramatically), but to re-integrate the processes of autonomous process-owners.

The following are generally cited in literature as reasons why re-engineering projects fail:

(i) Unclear definitions: re-engineering is more than automation or re-organization although it almost always effects organizational change. It goes beyond TQM seeking breakthrough measures of performance, pursuing multifaceted improvement goals, for instance quality, cost, flexibility, speed, accuracy and customer satisfaction - concurrently and with little trade off.

(ii) Unrealistic expectations: One of the consequences of (i) above, is the over-optimistic viewpoint about the domain of re-engineering.

(iii) Inadequate resources: Adequate resourcing of the re-engineering is a balanced mix of insiders and outsiders for the re-engineering. The question of IT resources also plays a part in this. 
(iv) Taking too long: Although it is generally contended that re-engineering projects may take anything from three to five years, few executives are that patient and few organizations can sustain themselves that long - particularly if the re-engineering is done from a reactive/preactive $^{3}$ perspective [11].

(v) Lack of sponsorship: In conjunction with (ii), re-engineering cannot be driven from a supply chain perspective, it needs buy-in from top management - more so since it generally entails culture changes.

(vi) Wrong scope: It is not possible to re-engineer an organization, it is only possible to re-engineer its processes, with many processes being inter-organizational and cross-functional. The likelihood of success diminishes if the scope of the re-engineering is restricted to certain processes only.

(vii) Mysticism: re-engineering is not a paradigm shift, it is an engineering discipline that enables transformation to take place.

(viii) Lack of effective methodology: Without some scientific approach, the re-engineering may consist of an AS IS without a proper TO BE scenario. One generic re-engineering model consists of four phases, namely analysis (AS IS), design (TO BE), transformation and evaluation [12].

(ix) Technocentricism: Note that generally, implementing IT (although radical by nature in terms of the applicable software delivery system) and implementing re-engineering are not the sametheir objectives being different. This (the first mentioned) may be termed the 'Keeping up with the Joneses effect' [12].

\subsection{The problem with the ubiquitous IT}

Whether re-engineering is called process innovation, business process redesign, business engineering or process engineering, organizations are trying to make radical and dynamic changes in the ways they operate. At the heart of re-engineering are two concepts, namely:

口 Organizations should view themselves in terms of processes (not functions, divisions or products) and

a organizations should think inductively instead of deductively.

The latter refers to the constant disruptions to the current ways in which they conduct their businesses. The proliferation of new IT is increasingly becoming a major contributor to this disruption. The table below summarises business rules that have become redundant through the power of IT.

\footnotetext{
${ }^{3}$ Reactive: Tendency to focus on the past; Pre-active: Maintaining the strategic fit by trying to predict the shape of the future and Proactive: Designing the future and making it happen [11].
} 
Table 2: Disruptive technologies

Rivi:

Information can appear in only one place at one time

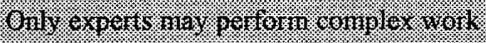

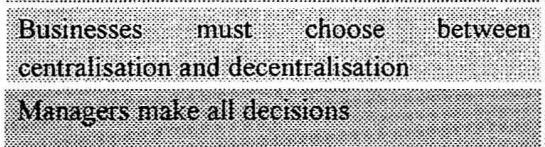

Field personnel need offices where they can recelve; store, retrieve, and transmit. information

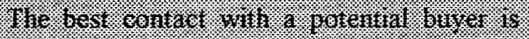

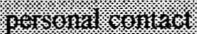

You bave to find out where things are

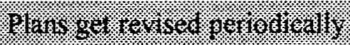

\section{MISR UP TIVE TI:}

\section{Shared database}

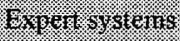

Advanced

telecommunications networks

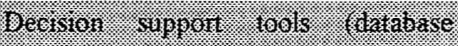

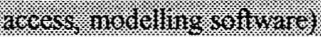

Wireless data communication and portable computers

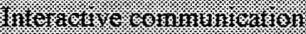

Automatic identification and tracking technology

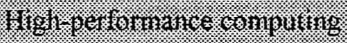

Source: [4]

Critics of re-engineering believe that the inherent problem of the concept lies not so much in IT's inability to do the re-engineering, as in the misleading label that in no way defines what it is, rather what it is not. They maintain: 'The case against re-engineering is continually handicapped by its unfortunate and technocratic label which does not in any way suggest what is it all about. The label itself is neutral, directionless, purposeless and therefore misleading. '[10: p105].

From the first CSC Foundation Index [8] studying the successes (or failures) of re-engineering, the most important theory to emerge (corresponding to other, similar studies) is that re-engineering is not enough on its own. It needs to be linked to technology. They pose the question: 'Why streamline a particular business when technology is about to render it obsolete?' Some IT gurus believe that the main problem with re-engineering lies in its unflinching focus on the bottom line - not on the IT [6]. They believe that redesigning processes as a cost-cutting measure is counterproductive as a lot of valuable knowledge and information tied up with these processes is simply discarded. Cowley subsequently decides that the shift towards re-engineering for growth is less an evolution of reengineering than a mask for its failures.

Geisler [13] contends that the re-engineering proposed by Hammer and Champy, cites IT as the enabling mechanism for organizations to reinvent themselves. The redesign of the work processes and the elimination of processes with little or no value added and the resultant overall redesign of the organization depend on the existence and support provided by ubiquitous IT [13]. Geisler believes that 
the fallacy in this contention is composed of the following dimensions that help explain the failure of re-engineering. These are:

(i) The information dimension

- Even the best, complete, timely, correct and clear information is not enough to fuel reengineering.

- If re-engineering is already flawed as a concept and major change programme, IT and the ubiquity of information cannot overcome these flaws.

(ii) The technology dimension

This is the growing evolution of IT from back room, cost-cutting efficiency purposes to frontend and strategic use for overall organizational performance, enabling organizations to advance on the learning curve, i.e. the evolution of IT from efficiency, to effectiveness, to transformational and strategic enhancement tool.

IT has helped create a boundarylessness between organizations. One such example is the principle of the holonic enterprise [14: pvii], following on the principle of holonics which they perceive comprising of the following elements:

1. Virtual, i.e. teamwork across boundaries of functional departments and/or across established companies (through IT)

2. Agile, i.e. the ability to rapidly respond, known as Turbo JT (through IT) and

3. Holonic, i.e. autonomous distributed systems (mainly through IT).

Dell Computers operating as virtual organization with their Direct Business Model [15], is probably the best example of such an organization. Dell realised the cost saving in putting their capital into those activities where they could add value to their customers, i.e. suppliers effectively become their partners in a virtually integrated business model. Technology is enabling co-ordination across organizational boundaries achieving new levels of efficiency and productivity, thus blurring the traditional boundaries in the value chain among suppliers, manufacturers and end-users. The net effect is that they are able to build and sustain relationships with their customers, which, in turn, creates valuable information, allowing them to leverage their relationship with both suppliers and customers. There is real-time information sharing (to the extent of shared databases and methodologies) and participation with supplier-partners, thus speeding time to market. Furthermore, keeping track of customer purchases, has generated valuable information that can substantially extend value back to the customers. Dell's success was brought about, not as much by re-engineering their processes as through virtual integration using information and IT and its effective substitution of information for inventory.

Thus, it is possible that IT is not the only catalyst and cannot act as such for re-engineering. It is suffering its own evolution. It certainly has brought about many changes in culture and the way work is performed, but it brings about its own form of change in the organization - one that does not necessarily correlate with the objectives of re-engineering. In view of this, Hammer and Champy's contention stated above could be seriously flawed.

\subsection{Re-engineering and Marxism}

At the heart of re-engineering lies the notion of discontinuous thinking - of breaking away from outdated rules and fundamental assumptions that underlie operations and of jumping the curve of the 
existing ways of doing business. Breakthrough performance improvements cannot be achieved unless there is a challenging of old assumptions and a shedding of the old rules that made the business underperform in the first place [2]. Every business is replete with implicit rules from earlier decades. These rules are based on assumptions about technology, people and organisational goals that are no longer valid. Thus, the re-engineering theory restates both aspects of Karl Marx's synthesis, namely revolution over evolution and holistic process over fragmentation [16].

The $19^{\text {th }}$ century social theories with their tenets of loyalty to roots in the past, historical development and gradual evolution, were considerably more realistic and tenable than the blue sky dreams of the revolutionary Utopians [15]. However, in a period rife with revolutions (for example, French, American and Napoleonic), this theory was considerably weakened by its inability to deal with momentous change. At this point in time, Karl Marx used Hegel's philosophy of dialectical materialism to synthesise social theory with some recognition of revolutionary change [17]. Marx called upon social revolutionaries to seek their organisational ideal as a potentiality already organically immanent from within, but antithetical to the existing organisation. They would subsequently make a revolution to emancipate that potential organisation from whatever obstacles prevented its realisation. Although Marx was mistaken in much of his economic and historical analysis, substituting 'business organisation' for 'society' and 're-engineering' for 'revolution', one can apply his maxims to modernday businesses.

When a business is lagging, gradual incremental (TQM-type) improvements will be insufficient to catch up with competitors and environmental changes. The second part of the Marxist synthesis is the organic character of the process that the revolution is to emancipate. According to re-engineering theorists, re-engineers are to hunt for potential processes to re-engineer within the fragmented activities of modern organisations. They must understand these processes without obscuring their identity with an analysis of their disjointed parts. Finally they will replace fragmented tasks with holistic processes that integrate values, goals and customer needs, along with the nascent ability to satisfy them. This is a recurrent theme in Hammer and Champy's book [2,4]. These authors identify a need to overturn the division of labour, which underlays the increased productivity of industrial economics. According to them (Hammer and Champy), the fragmentation of business processes (which worked well when processes were relatively simple and without the need for complex integration) is inadequate in a world of intense competition, geometrically accelerated rate of change and customer self-awareness. In this, their theory is as revolutionary as Marx's, although the revolution they preach, is a revolution from above. Serving as a sort of central nervous system, new information and communications technologies permit organisations to retain centralised intellectual control over resources and processes, while benefiting from the increased flexibility and customisation inherent in physical decentralisation.

Finally, organic business processes have personalities - they are composed of people, having different values, needs and goals. The re-engineering/revolutionary tone is set by the authors when they consistently invoke violence and revolution in rhetoric and practice. Hammer and Champy's dogmatic pronouncements resonate with radical views put forward by other revolutionaries like Robespierre, Lenin, Mao and Marx. Some authors warn that by replacing some of Hammer and Champy's nouns, it is possible to produce slogans attributed to those who gained power by overthrowing the existing order. 
It is unlikely that the most widely read book on re-engineering [2, 4] (carrying the subtitle 'A Manifesto for Business Revolution' and claiming to be a seminal book comparable to Adam Smith's 'An inquiry into the nature and causes of the wealth of nations' - the intellectual underpinning of capitalism) can successfully spread the premise that the only way to improve processes (or capitalism), is to obliterate them - radically, fundamentally and dramatically.

\subsection{The promise of the perfect solution through radical change}

There is a growing concern that re-engineering is another attempt, usually from top management, to impose a new structure over the old and to take one set of rules and impose them on the rest of the organization. It presupposes that one can design a perfect solution, whereas the 'machine' will subsequently comply with the new set of instructions. With re-engineering, there is little attempt to institute ongoing workable processes for creating positive change once the radical change (of the business or its processes) has been introduced, and, moreover, failed, making it critical to pose the following important questions:

a What happens when the organization needs to change again?

口 What if the re-engineering does not deliver its promises?

This paper will address the above by questioning a solution (e.g. re-engineering) that is based on the premise of Newton's law- abiding, cause and effect view of the world, more so if it provides a once off fixed solution and subsequently continue to introduce the de-engineering solution as clean up in the wake of re-engineering's failure to deliver.

\section{NEWTONIAN BUSINESS SCIENCE}

Newtonian science, the underpinning of civilisation from the 1700 s to the present, is rooted in physics and mathematics - rule-bound disciplines that require data up front in order to operate. The core of the paradigm, namely Newton's laws of motion (in which he reduces all motion to three simple laws), suggests that the world is a well-behaved machine. It offers the promise of a well-behaving and predictable universe. It underscores the belief that relationships between cause and effect are simple, clear, predictable and linear.

This '..if $\mathrm{X}$, then $\mathrm{Y}$ follows ..' (cause and effect) view of the world prevailed for almost three centuries, delighting the scientists whose primary objectives were to predict and control. With the advent of the Industrial Era, Newtonian science led us to focus on businesses from a stable system perspective. Whenever some force would upset the system, it would be the leadership's duty to reestablish equilibrium. Not to do so would constitute failure. With stability as the ultimate goal, the paradigm implied that order should be imposed from the top (the top down, command-and-control leadership style) together with supporting structures for decision-makers (resulting in bureaucracies and hierarchies). These were the cornerstones of the Scientific Management movement, having as its building blocks regularity, predictability and efficiency.

\section{BEYOND LINEAR THINKING - THE CHAOS PARADIGM}

It is evident that existing organizational models are no longer delivering the promise of competitive and sustainable change, arguably since organizational life is designed from images of the Newtonian 
universe. We manage by separating things into parts, we believe that influence occurs as a direct result of forces exerted from one person to another. We engage in complex planning for a world that we continue to expect to behave predictably. Moreover, we continually search for better methods of objectively perceiving the world. Intentionally or not, we work from a world view derived from the natural sciences. However, the sciences have changed and we should at least expand our search for organizational principles to the modern sciences of our times.

Consider the dissipation of structures, whereby a system loses its present form so that it could reemerge in a form better suited to the demands of the current environment. Based on the work of 1977 Nobel prize winner, Ilya Prigogine ${ }^{4}[18]$, one can conclude that, in a dissipative structure, things in the environment that disturb the system's equilibrium play a crucial role in creating new forms of order [19]. As the environment becomes more complex, generating new and different information, it provokes the system into response. New information enters the system as small variations from the norm. Information continues to grow - finally to such a level of noise that it can no longer be ignored by the system and thus, falls apart. However, generally, the system reconfigures itself at another level of complexity - one more suitable to handle the new environment. Thus, these systems show that disorder can be a source of order and that growth is found in disequilibrium and not in stability. Moreover, destabilizing organizational issues (e.g. fluctuations, disturbance and imbalances) should be viewed as primary sources of creativity rather than impending disorder bent on destroying the system. Scientists believe that there is order out of chaos and that, throughout the universe, order exists within disorder and disorder within order. This leads to the principles of chaos theory.

Like all buzz words, the concept of chaos does not follow the same connotation it would in everyday use. Stewart [20: p16-17] cites two definitions for chaos theory: 'Complete disorder, utter confusion' and: 'stochastic behaviour occurring in a deterministic system' - the latter indicating something like a lawless behaviour governed entirely by law. Although it incorporates elements of chance, chaos is not random disorder. Rather, it attempts to understand the behaviour of systems that do not follow a linear pattern or show conventional cause and effect over time. When viewed as a whole, these systems exhibit definite patterns and structures. However, at no single point can their future behaviour be predicted from the past. In this sense, chaos is far from random and therefore chaotic systems can be both determinate and unpredictable. These systems can organise and renew themselves, with periods of order broken by sudden transformations in a direction that has elements of chance and cannot be reversed.

The main characteristics of chaos theory are [21]:

(i) Non-linearity: Chaos theory proclaims that (even minuscule) changes in a system's initial conditions could amplify in their effects which could have little resemblance to the beginning. This makes anything beyond short-term predictions impossible.

(ii) Feedback: Chaotic systems evolve from accumulated feedback acting like an iterative process. Output at every step in the system provides material for a new formulation and new outcome,

\footnotetext{
${ }^{4}$ Prigogine's work focuses on feedbacks insofar as they explain spontaneously emerging structures stimulated by energy input from outside and dissipative structures based on far-from-equilibrium thermodynamics.
} 
thereby amplifying deviation and working to destabilise existing states and introduce new patterns of behaviour.

(iii) Bifurcations and phase changes: Destabilising the system can lead to new directions, character or structures - called bifurcations. These are points where, because of a catastrophic leap, the system arranges itself around a new (different) underlying order. Although the occurrence of bifurcations can be predicted, their outcomes cannot.

(iv) Strange attractors: Chaotic systems have strange attractors whereby outcomes wander constantly and unpredictably within a bounded range. Maps of such situations in which multiple variables are pulling events in contradictory directions, resemble the scribbled doughnut or butterfly wings familiar to mathematicians. Still, the underlying order represented by the attractor, limits excessively erratic behaviour and imposes structure - even though unpredictability may exist within the bounds of that structure.

(v) Scale: Since the evolution of a chaotic system is vastly complex and also prone to disturbance by chance, it is impossible to determine its underlying pattern by looking at a single event. Interpretation of the form and coherence of a chaotic phenomenon is affected by the scale used to measure and view it. Thus knowledge of the full map (or history) is important since different behavioural patterns can prevail in different parts of the map. Chaos theory maintains that one should see the whole (not the parts) to make decisions.

(vi) Fractals and correspondences: Following on the previous notion, chaos theory assumes that concentrating on individual units yields different or misleading information. This holistic view in chaos theory differs sharply from the Scientific one that believes that the behaviour of microcosms can be used to deduce that of the whole system. In a chaotic system, a strange attractor is a fractal curve that imbues all the diverse elements it governs with its own underlying pattern. Thus a fractal representation of a system shows very similar, though not identical patterns at successively greater magnification. This makes it possible to analyse chaotic systems by tracking similar patterns through successive stages of evolution.

(vii) Self-organisation and self-renewal: The ability to reorganise is inherent in the chaotic system itself and does not require any external intervention. Some theorists believe that this sensitivity of a chaotic system to its own history enables it to pull out of disarray - as well as impelling it into chaos. Thus, chaos follows an inner logic on the one hand and has continuity on the other, because of its iterative nature. Thus, a chaotic system is an unstable combination of randomness and plan, broken by flashes of change.

Chaos science therefore highlights the role of chance, the possibility of many outcomes and the ability of the observer to choose which outcome will be called reality. It is emancipatory, with the price for open-endedness being extreme uncertainty and the loss of a sense of control. In a business sense, chaos theory could explain diversity theories (for instance, feminism or racism). It offers not so much novel solutions as a structure for persistent problems and new models, since it emphasises uncertainty, openendedness, plurality and change. In this it runs counter to the goal-oriented, certainty-seeking mode of the Industrial Age.

Chaos, as referred to in the sciences, is a revolution in the understanding of the way the world works. Its revelations have overturned Newton's law-abiding universe and replaced it with a world of infinite 
complexity in which everything is connected in a vast, ever-evolving web. Chaos in a business sense does not infer collapse of the organisation and its structures, rather it refers to the eminently orderly disorder that mystics for centuries (and scientists fairly recently) have recognised as nature's way. Wheatley [19: p21]: 'Throughout the universe, then, order exists within order and disorder within order. We have always thought that disorder was the absence of the true state of order.' And concludes: 'While we have lusted for order in organizations, we have failed to understand its true nature.'

The question is whether chaos theory can provide businesses today with the necessary methods and metaphors to deal with the shifting paradigm of work. Does its self-organising capabilities lend themselves to the self-organisation of people or processes? Is there utility in chaos theory which could add to (even supplant) Newton's laws?

While chaos theory offers few practical guidelines, three caveats about intervention within a highly unstable business environment may be highlighted [21]:

a Change has to evolve from within, it cannot be imposed from the outside.

a Intervention works most efficiently at critical points, when the system is well on the road to instability (the 'burning platform effect').

- There should be quick response and actions at these crisis points, otherwise the system may take on its own shape - which might not necessarily be congenial to the organisation.

According to the chaos paradigm, the organisation should not attempt to control existing attractors but should rather fit into them. On the other hand, accepting the metaphors of the chaos paradigm in no way negates completely the models of Newtonian science. Either approach can be appropriate, depending on whether the system behaves predictably or not. At this stage, however, chaos theory is more useful as an analogy than a source of practical solutions for relationships between organisations and their environments. It helps in structuring persistent problem situations where there is insufficient knowledge about cause and effect relations and where leadership is able to act in unpredictable ways. In this sense, chaos theory provides rules as definite as the Newtonian ones it challenges. The new rules (pluralism, change and chance) may bring some comfort to leadership. However, in many situations, chaos theory balances out the overly rational management styles and approaches in situations where pro-activity cannot prevail. It provides a useful antidote to overly rigorous linear views without being a fully satisfactory replacement.

\section{DE-ENGINEERING THE CORPORATION}

\subsection{Beyond re-engineering}

Following on the discussions earlier on re-engineering's failures, Figure 1, although not clear in futuristic scope, attempts to show that re-engineering is by no means deemed the ultimate in business enhancement tools. It forms part of a vast and broad band of evolutionary tools and techniques, each serving the forces of its time, each creating the pathways towards its own destruction. In this paper, the notion of de-engineering (as form of chaos management - because of the environment and because of the failed re-engineering) is introduced as some 'yet-to-come' (YTC) methodology over re- 
engineering, to fill the gap left by re-engineering because of the latter's Newtonian approach to business problems and to the environment.

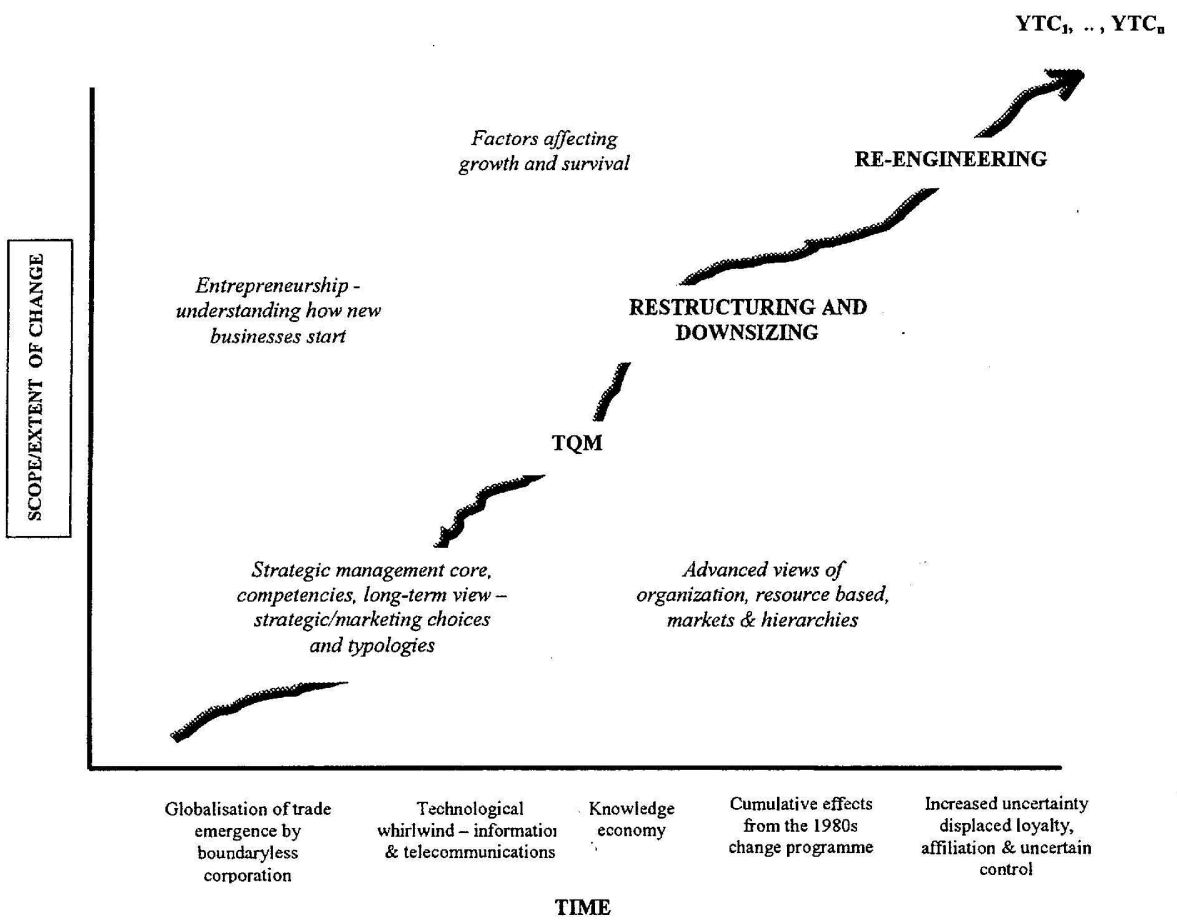

Figure 1: An evolutionary view of the new business world and important business enhancement programmes $\left(Y T C_{i}=\right.$ programmes as yet not identified $)$

Source: [13]

Geisler [13] contends that re-engineering is not so much a paradigm shift as an obsolete promise of a solution to a set of questions, that was sold to management as a manifesto for change [2] - perhaps ahead of its time on the evolutionary scale of management knowledge, since it had few knowledge tools available to tackle these problems.

In conclusion, the 'popularity' of re-engineering is a signal that organisations perceive the need for improved performance. From Hammer's perspective [5], this entails an on-going process rather than a once-off cure. Thus, it is important to implement the re-engineering with the minimum scope for failure. Most authors maintain that in order for re-engineering to be a success, the strategic relevance 
of a specific process must be assessed and the culture qualified, both of which are combined in the above model. With research pointing to the many failures of re-engineering implementations, it is important for organisations to address the cultural imperative as one of the key variables in the implementation of radical change. Thus, the individual and combined impact of culture and strategic relevance could resolve some of the re-engineering challenges that organisations are facing today.

\subsection{De-engineering the corporation}

Wheatley [19: p20] contends: 'Re-engineering is the supernova of our old approaches to organisational change, the last gasp of efforts that have consistently failed.' She adds: 'Re-engineering is the biggest and most dramatic bandwagon that has hit the business and organisational world in a long time."

She agrees that it is necessary to fundamentally redesign bureaucratic organisations, but that the net effect is a string of failed change efforts over the years. There is a growing concern in the literature on the subject that re-engineering is another attempt, usually from top management, to impose a new structure over the old and to take one set of rules and impose them on the rest of the organisation. It promises the existence of a perfect solution. The question remains: What happens when the organisation needs to change again? One asks this because with re-engineering there is little attempt to institute ongoing workable processes for creating positive change. With de-engineering, the question shifts to 'Has the organisation's capacity to change increased and improved? Have we developed an organisation that can continue to be responsive and adaptive or have we created a new structure that will atrophy as the environment shifts?' [19: p20]. By its very definition, the term de-engineering, implies a self-organising pattern for leaders and workers.

The de-engineering phenomenon then is built on the premise that there is natural order and that patterns do exist, arising without any management intervention and without any pre-engineered design. The contention is that any programme of change that tries to impose a structure on everyone, works against people's natural tendencies (and without their involvement). People have a natural tendency to create order as needed - provided that certain conditions are present. In this context, the two major resources of organisations (the people and the information) need to work coherently. Organisations need to merge the science of management and leadership with the modern studies of complexity so that people may work in an information rich environment.

Thus, de-engineering entails a new definition of leadership, where the traditional leader may not even be present during a crisis and the role of the chaordic (=from cha-os to or-der [12]) leader prevails. The challenge is to move information through the organisation without knowing ahead of time who will need what or where it may be needed. In this context, it is important that organisations clearly define what the organisation is trying to achieve and how people should behave, given a particular situation. Thus, from the notions of chaos, it is possible to create well-ordered and efficient organisations that will be able to constantly change their physical structure, by creating an awareness of the creation of conditions for the order of the organisation to emerge and change. This is the new challenge: Order and answers do not come from consultants, management programmes or the external environment; people are able to create the answers and the order needed, provided that there is available, accessible 
and timeous information and that decisions can be made at the local level based upon a strong sense of organisation identity. It is suggested that these points make organisations truly agile, adaptive, versatile and resilient.

The difference between this approach and re-engineering lies in the fact that re-engineering assumes that the solution for failing organisations will come from some group of experts or consultants, whereas, de-engineering supposes that the organisation has access to its own intelligence and that conditions exist that support the use of that intelligence. Within this proposition, it is possible for the organisation to change continuously and organisations become living entities rather than well-tuned machines. In de-engineering the supposition is that people are involved - not only the re-engineering teams. In fact there is a meaningful involvement of the entire organisational force. It is still important to fundamentally redesign how organisations do their work, thus not only radically changing the organisation's structures, but also creating an organisation capable of and committed to a next round of change. It follows that there is a new framework of understanding businesses in the light of the problems they face.

It is interesting to note that problems often crop up seemingly spontaneously in widely separated places or arise in several disciplines at once (synchronicity ${ }^{5}$ ). For example, at about the same time that Darwin proposed his evolution theory, Alfred Russel Wallace in Malaysia published similar ideas. At the time that the $16^{\text {th }}$ century Dutch school of painters were drawing light for its effects on interior spaces, depicting how light became transformed through coloured glass, Newton was studying prisms of light and its behaviour as it passed through small apertures. Recently there have been similar parallel concepts between science and business. Businesses began slowly to engage in a world of connectivity through electronic networks at the same time that quantum physicists began earnestly to explore the notions of cosmic interconnectivity. Scientists and business people use surprisingly similar language to describe the new world of interconnectivity. Research in both fields revolves around contributions to 'growth and vitality'. The language of both has converged around 'partnerships', 'interrelationships' and 'mutual commitments'. Emergent thinking about organisations focuses on their abilities to selfrenew and self-organise.

However, it is contended that business (and social) scientists have increasingly endeavoured to be rigidly scientific and mathematical in their appraisal of business problems and subsequent solutions, whereas pure scientists have moved along to describing new realities. The net effect of the first construct is a rigid management theory, scientific appraisals of constraints and weaknesses which, in part, led to the notions of 're-engineering', 'restructuring', 'downsizing' and 'redesigning' to name but a few. With one of the guiding principles of nature being that at all levels, nature resembles itself, this parsimony of nature's laws is indicative of the following important consideration: If nature uses certain principles to create her infinite diversity, it is highly probable that those principles could (or should) also apply to business organisations.

For instance, it is likely that the movement towards participation is rooted in the changing perceptions of the organising principles of the universe as defined in quantum mechanics. Along with participation

${ }^{5}$ Regarding the synchronicity across the boundaries of the sciences, refer Russel, 1979 or Boring, 1950. 
and leadership, the business world is confronted by information as the new (and basic) ingredient (and requirement) of the universe. Information is largely intangible, transcending time and space. It does not have to obey the normal laws of matter and energy and can assume form or communicate instantaneously anywhere and anytime in the information realms of society. In a business context, the problem is not only information overload (which technology should be able to address), it is the inexorable movement towards a new paradigm where information (generated and exchanged) will determine the future. Failure to recognise the generative properties of information will inhibit organisations and render them unable to manage.

Emerging from the constant flux, is a state of global stability whereby incremental movements merge into a whole that can resist most of the demands for change at global level. The motion that keeps all systems in harmony will be that of self-reference and self-renewal, thus replacing Newton's mechanistic regulated world. Thus, it is contended that from chaos and complexity comes a new notion of simplicity over revolution and re-engineering. In quantum mechanics, the world ceases to be a mere machine, finite or discrete. It describes de-engineering (evolutionary by definition) as more than a solution, rather a replacement for re-engineering (revolutionary by definition), when it is contended that Most of the other steps in our understanding of nature were really evolutionary in that they sprang from previously established foundations: facts were reorganised or connected in new ways, or seen in a different context. Quantum theory, however, broke away completely from those foundations; it dove right off the end. It could not (cannot) adequately be described in metaphors borrowed from our previous view of reality because many of those methaphors no longer apply. But the net result has not been to obscure reality or make the nature of things more elusive and murky. On the contrary, most physicists would agree that what quantum theory has brought to science is exactly the opposite - concreteness and clarity.' [24: p106].

\subsection{De-engineering as paradigm shift}

When a paradigm shifts, it affects the way people look at the world and the way they understand it, so that patterns and logic are totally new and the existing pattern and logic are no longer valid. The shift follows from the emergence of new questions. For instance, Instead of 'What type of factory should we build?', one would ask: 'What business should we be in?' This provides a new perspective, with totally new views of what the world is like and how and why things seem as they are, and also what work they do. Clark [22] provides an excellent example of this in his treatise on the development of Western civilisation. He focused on the year 1100 when radical changes occurred in European culture, architecture, sculpture and the people themselves. Clark notes that these changes occurred within one lifetime - not as a result of some technological breakthrough, but rather through a fortuitous release of energy and a leap to a higher plane (or new wave). Real shifts in scientific paradigms appear as the whirlwind activity of some great scientist/revolutionary (like Newton) who redesigns the world in his head and leads it in a new direction. Thus, radical change in management occurs as a happenstance combination of a leap in technology and a (possibly subsequent) shift in management technology, outlook and perspective. Such changes may be due to environmental turbulence in a manner similar to the great discontinuities in biological evolution attributable to major changes in the earth's environment (like the end of the Ice Age). The merging of the technology leap with changes in management philosophy changes do not generally occur all at once. There is generally a gap between the occurrence 
of these two dimensions of the radical shift in management paradigm. Shifts in management paradigm are complex and lengthy and seldom attributable to a single innovator. They are incremental rather than sudden. On the other hand, the triggering dimensions of technology and change in management philosophy come from outside the organisation and its leadership. Management scientists digest potential changes and transmogrify them into a long-term indoctrination effort which results in shifts in paradigm. There are Gurus in management (like Deming, Champy and Hammer, Drucker and Toffler), there are no Newtons, Einsteins or Mandelbrots. Knowledge in management and organisations is still merely a fragile assortment of methodologies and findings borrowed from other disciplines like the sciences. The integrated framework is still in its infancy, made more complex by the array of questions that have arisen and management's inability to field or formulate simplistic yet encompassing models of how organisations exist. Moreover, at any given time, there is not only one, but several paradigms prevailing in the management and organisational discipline - some of which are completely divergent or paradoxical.

In this paper, de-engineering is introduced as paradigm shift from Hammer and Champy's reengineering radical change methodology of the 1990s [2, 4]. It does not ignore the fact that organizations need radical change in order to survive, rather it proposes a solution on top reengineering, one so radical as to be analogous to a paradigm shift and one that goes beyond reengineering and tries to provide new alternatives when re-engineering has failed. In this, Figure 1 summarises past and future enhancement programmes (YTC) of which de-engineering may be one.

\section{CONSEQUENCES OF DE-ENGINEERING}

\subsection{The end of a machine-like organization}

Seventeenth century organizational structures are starting to crumble where organizations survived through planning, predicting and analysis of the environment. There existed an unwavering belief in cause and effect. In Newton's machine-like universe, we were assured of determinism and prediction, causing us to organize our work and knowledge to fit this universe. Some examples of organizations conforming to the machine image of the Newtonian view are [19]:

ㅁ People are organized into functions, roles and accountabilities - drawing lines of authority and limiting responsibility;

- Organizational charts depict the detailed working of the 'machine';

口 The concept of organizational fit plays an important role if restructuring/re-engineering/strategic planning;

․ Organizational theory focuses on a 'reduction into parts', 'the proliferation of separations' and the notion that, in studying the parts, one could arrive at knowledge of the whole, thus drawing the world in lines and boxes;

- Knowledge is divided into disciplines and subjects;

- Organizations focus their attention on structure and organizational design;

- Organizations concentrate on gathering extensive numerical (current and historical) data and making decisions using financial ratios;

口 Information is portrayed in charts that divide our viewpoint (e.g. market share, employee count, customer ratings);

a Power is measured in terms of ".. our share of the pie'. 


\subsection{Organizational behaviour in the quantum world}

Although we believe that information has created a kind of societal boundarylessness, this is difficult to attain as organizations are generally bounded and defined. Since the twentieth century, it has become apparent that the modern world cannot be explained by Newton's laws - a new and different science is required to explain new phenomena. Moreover, Cole [24: p106] writes: 'Most of the other giant steps in our understanding of nature were really evolutionary in that they sprung from previously established foundations'. Thus, the world ceases to be a machine and is viewed rather as a dynamic, living force. Absolute prediction and uniformity are impossible. People become different persons in different places and at different times. Moreover, following from Heisenberg's Uncertainty Principle ${ }^{6}$, also known as the principle of quantum mechanics, we can measure position or we can measure momentum, but not both at the same time. In organizational terms, this so-called duality spells the end to any deterministic, quantifiable universe. Moreover, the act of observation causes the wave to collapse, inferring a relational universe. Thus, our acts of observation are part of the process that brings forth the manifestation of what we are observing. Thus, at the moment that the wave function collapses, Newtonian physics supersedes the quantum phenomena.

\subsection{Maintaining a state of disequilibrium through de-engineering}

By nature, organizations behave towards equilibrium seeking modes of state in accordance with the Second law of thermodynamics ${ }^{7}$ [26]. According to the Oxford dictionary [27], equilibrium is: '.. $a$ condition in which all acting influences are cancelled by others resulting in a stable, balanced or unchanging system, the condition of a system in which the resultant of all acting forces are zero.' In classical thermodynamics, equilibrium is the end state in the evolution of isolated systems, i.e. that point at which the system has exhausted all of its capacity to change. In other words, at equilibrium, there is nothing left for the system to accomplish. However, living, open systems (like organizations), do not seek equilibrium, rather they import energy from the environment and continuously maintain a state of disequilibrium. In fact, '.. form and function engage in a fluid process where the system may maintain itself in its present form or evolve to a new order' [19: p91]. Thus the system is not locked into a specific form or solution, but has the natural capacity for new structures, depending on its needs. Thus, these adaptive organizations have the capacity to self-organize/renew, avoiding rigid structures or fixed solutions (analogous to Prirogine's dissipative structures [18]).

Information becomes the medium of the organization and acts as nutrient [28] in that: no organization can exist without it; the organization feeds off it and it has to be everywhere in the organization in order to sustain the organization. Only when there is sharing of information and it belongs to everyone, can people organize rapidly and effectively around shifts in customers, competitors or the environment. People need access to information that no-one could predict that they may need until they need it. The broad availability of information does not mean that all decisions move to local levels. It provides the organization with a systems perspective - a notion that more members of the organization should 'inform' available data for effective self-organization to occur. It is information (unplanned, uncontrolled,

\footnotetext{
${ }^{6}$ There are features of the universe that cannot be known with complete precision. Such uncertain aspects would become more severe as the distance and time scales become smaller. Measurements cannot be done for time and space simultaneousiy. [refer Greene, 1999: 424 or Khalil, 1997]

${ }^{7}$ Also known as the law of entropy. Describes the equilibrium, end state of a structure which arises from the chaotic interaction of the molecules which make up the structure.
} 
abundant and superfluous) that creates the conditions for the emergence of fast, well-integrated and effective responses.

Complex, living systems thrive on information-processing and the constantly changing edge between stability and chaos (also known as the edge of chaos [28]). It is possible for new information to enter, while the system retains its identity. The implosion of IBM and General Motors is evidence of how sophisticated IT and measurement systems can create a sense of internal order while failing to take cognizance of critical new information.

This new notion of self-organisation makes structures and solutions temporary. Resources and people come together to: Create new initiatives; respond to new regulations and shift the organisation's processes. Leaders emerge at and from the needs of the moment. One surprising consequence of organisational complexity is that few of the sophisticated movements are directed by specific leadership [28]. They maintain that there is never any rule about a leader or direction. Rules focus on individual behaviour in relation to other employees. Nature abounds with examples of similar structures. Social insects, bird flocks, fish schools, human traffic jams, all exhibit well-synchronised and highly ordered behaviours which are not directed by any leader, but instead focus around a few rules at the local level. The above notions go against the inherent Western beliefs regarding planning and authority, and will be difficult to implement, bearing in mind the entrenched Western cultures and philosophies. There are fewer levels of management structures. Experimentation is the norm. Local solutions predominate but are kept local and are not elevated to a model for the whole organisation. Involvement and participation constantly deepen. These organisations are experts at the process of change. Employees understand that their organisation is a process of continuous organising. Descartes saw the world as a clock-like machine and subsequently built an entire philosophy around this metaphor. The principle tenets of this lifeless and mechanistic world (these being dominance and control, reductionism, determinism and materialism) provided the foundation from which modern social and business institutions evolved. The result was fragmentation and alienation [29] - for which re-engineering offers some form of solution, even if not complete. The principle of re-engineering the processes is not sufficient for quantum leap change. One reason for this is that the traditional business organisation is not designed for change, but rather for stability and the creation of predictable and certain results. Within the stable environment of the previous wave, this proved satisfactory. However, it is almost impossible for these solid and immobile blocks of stone to accept large changes as would be required through advancing technologies and the re-engineering concept. Thus, it is hereby proposed that the first re-engineering should not be in terms of the business processes (and subsequently, the business itself), but in terms of the organisation itself - essentially because it is designed for stability.

The need for change is constant and unavoidable, thus the fundamental nature and shape of the organisation should be transformed to make future changes easier. These organisations could typically behave according to the following patterns: 
- Employees collectively determine the direction and subsequently empower leadership to point the way.

a Leadership could help (collaborative) teams to realise they are off course and assist in the realignment within the whole.

- Frontline employees would be responsible for the movement of the organisation, not the managers.

Thus, leadership is challenged to assist in the co-ordination of activities and the clarification of the organisation's status and direction and not to carry the burden of responsibility for the whole organisation.

Lastly, in creating the de-engineered organisation, one should consider alignment between all elements within the organisational ecosystem, these being: culture, structure, technology, process and shared vision and their interconnectivities. There is a somewhat paradoxical need for freedom and order, i.e. autonomy at the local level - Jantsch [30: p40] writes: 'the more the freedom in self-organization, the more the order'.

\section{CONCLUSION}

Technology is dramatically and significantly altering the ways in which society is conducting its affairs and the way in which the world is preparing for the future. Consequently, changes bring about their own challenges and opportunities. Moreover, these new dimensions bring about dramatic business transformation - the object of this, being to control the challenges and take advantages of new opportunities.

Success in an increasingly competitive global environment is constantly being explored by leadership and their organisations. Traditional barriers such as national boundaries, currencies, regulation, a strong workforce and economies of scale that once sheltered businesses are crumbling and will, in all probability, disappear. This increases challenges and opportunities for businesses. Leaders making critical business decisions by applying traditional ('old') methods will not achieve the successes that they aspire to. Competition within this new paradigm requires new rules, policies, structures, roles, leadership and organisation. It demands a radical transformation of business itself, of its processes, its leadership and its resources. The significance of business change is expanded and related to the evolution and growth in the fields of technology and, specifically, information technology. The strange and exciting phenomenon of self-organisation is explored and largely became the focus of this paper. The point was made that chaos theory stresses ultimately reaching some (new) point of equilibrium, this following on the self-organising ability of the chaotic system. Subsequently, the notion of the selforganisation as a form of re-engineering, called de-engineering, was introduced. With re-engineering reporting many (and costly failures) it is proper to finally suggest a case for 'de-engineering' the corporation (or cleaning up after re-engineering).

The value of the self-organising phenomenon becomes more relevant insofar as de-engineering is proposed to counter the re-engineering failures and misconceptions. The uses of chaos and its role in de-engineering becomes necessary and evident to enhance the performance of organisations especially after re-engineering. The principle of self-organisation directs organisations to become more 
focused on letting information take its own course rather than developing new models or frameworks or employing existing ones. The principle of self-organisation presupposes no organised starting point, it lets the organisation move into confusion after which the information or organisation will, of its own accord, crystallise into new and exciting forms and ideas.

\section{REFERENCES}

[1] Toffler, A. 1980. The Third Wave. London: Pan Books.

[2] Hammer, M. and Champy, J. 1990. Reengineering the Corporation: A manifesto for Revolution. London: Nicholas Brealey Publishing.

[3] Hamel, G. and Prahalad, C.K. 1994. Competing for the future : Breakthrough strategies for control of your industry and creating markets of tomorrow. Boston Mass: Harvard Business School Press.

[4] Hammer, M and Champy, J. 1993. Reengineering the Corporation: A manifesto for business revolution. London: Nicholas Brealy Publishing.

[5] Hammer, M. 1995. The Reengineering Revolution: A handbook. New York: Harper Business.

[6] Cowley, A. 1995. PC Week. 22 May, (20): E2(2).22.

[7] CSC FOUNDATION INDEX. 1998. Information systems at work in business reengineering. Information week, 10/23/98, Issue 550, p69.

[8] CSC FOUNDATION INDEX. 1994. Re-engineering reviewed. Economist, 7 February, 332 (7870): $\mathrm{p} 66$.

[9] Moser, H.W. 1996. Short takes. Journal of Business strategy. May/June.

[10] Zeleny, M.1995. Editorial. Reengineering. Human Systems Management, 14: 105 -108.

[11] Ackoff, R. 1981. Creating the corporate future. New York: John Wiley.

[12] Pellissier, R. 1999. The Impact of information technology on business organizations of the future. Thesis in partial fulfillment of the $\mathrm{PhD}$ in the Department of Industrial and Systems Engineering.

[13] Geisler, E. 1997. Managing in the aftermath of radical corporate change: Reengineering, restructuring and reinvention. Westport: Quorum Books.

[14] McHugh, P, Merli, G and Wheeler III, W.A. 1995. Beyond Business Process Re-engineering: Towards the holonic enterprise. New York: John Wiley. 
[15] Magretta, J. 1998. The power of virtual integration: An interview with Dell Computer's Michael Dell. Harvard Business Review, March-April, v 76, n2 p72(13).

[16] Sanders, R.L. 1997. If Marx had been a business process reengineer. Records Management Quarterly, 31(2): 58-65.

[17] Russel, B. 1979. History of the Western Philosophy. London: Unwin Paperbacks.

[18] Prigogine, I. 1978. Time, structure and fluctuations. Science, 1 September. 201: 4358, $777-$ 785.

[19] Wheatley, M.J. 1994. Leadership and the new science. USA: Berrett-Koehler Publishers.

[20] Stewart, I. 1989. Does God play dice? Great Britain: Basil Blackwell Inc.

[21] Murphy, P. 1996. Chaos Theory as a model for managing Issues and Crises. Public Relations Review, Summer, 22(2): 95-113.

[22] Clark, K. 1969. Civilisation: A Personal View. New York: Harper and Row.

[23] Boring, E.G. 1950. A history of experimental Psychology. Englewood: Prentice-Hall, $2^{\text {nd }}$ Edition.

[24] Cole, K.C. 1985. Sympathetic vibrations: Reflections on Physics as a way of life. New York: Bantam Books.

[25] Greene, B. 1999. The elegant universe. London: Jonathan Cape.

[26] Khalil, E.L. 1997. Chaos theory versus Heisenberg's uncertainty: Risk, uncertainty and economic theory. American Economist. 41(2): 27-40. Fall.

[27] The Oxford dictionary for the business world. Oxford; Oxford University Press. 1993.

[28] Wheatley, M.J. and Kellner-Rogers, M. 1996. The Irresistible Future Organisation. Strategy and Leadership, July/Aug, 24(4): 18-24.

[29] Youngblood, M.D. 1997. Life at the edge of chaos: Creating the quantum organization. Perceval Publishing.

[30] Jantsch, E. 1980. The self-organizing universe. Oxford: Pergamon Press. 